\title{
Effect of Suture Tubularization on Quadruple Stranded Hamstring ACL Grafts with Femoral Suspensory Fixation: A Biomechanical Study
}

\author{
Matthew Richard Moralle ${ }^{1 *}$, Mark DeCotiis ${ }^{1}$, Linda Chen ${ }^{2}$, Balazs Galdi ${ }^{1}$ and Jessica Cottrell ${ }^{3}$ \\ ${ }^{1}$ Department of Orthopaedics, USA \\ ${ }^{2}$ Hospital for Special Surgery, New York, USA \\ ${ }^{3}$ Department of Biological Sciences, USA
}

*Corresponding author:Matthew Richard Moralle, Department of Orthopaedics, Rutgers New Jersey Medical School, 140 Bergen St., Suite D-1610Newark NJ 07103

Submission: 钢 March 12, 2018; Published: 眥 April 04, 2018

\begin{abstract}
Anterior cruciate ligament reconstruction success is dependent on several factors including fixation strength, anatomical placement, and the mechanical properties of the graft tissue. Currently it is unclear if tubularization of a quadruple hamstring graft is an effective way to increase its tensile strength. The purpose of this study was to quantify the impact of whip stitching as a form of suture tubularization on the mechanical properties of quadruple-strand hamstring anterior cruciate ligament reconstruction using a femoral suspensory fixation. This study utilized bovine hamstring grafts, prepared as quadruple-strand hamstrings with and without tubularization. Each graft type was tested using a synthetic bone block model and a porcine tibia model to determine ultimate load, yield load, stiffness, and graft displacement at failure. The synthetic bone block model demonstrated that grafts with tubularization had statistically higher yield load then grafts without tubularization. The porcine model demonstrated that the ultimate load and yield load were found to be statistically similar between the graft groups but the tubularized group had greater stiffness when compared to untubularized grafts. In conclusion, our data shows that the effect of tubularization on a quadruple-strand hamstring graft with a femoral suspensory device positively effects the mechanical properties of the graft.
\end{abstract}

\section{Introduction}

Approximately 100,000 anterior cruciate ligament reconstructive surgeries occur every year in the United States. Of these surgeries, 3,000 to 10,000 ACL reconstructions will end in graft failure [1]. The success of ACL reconstruction depends on many factors including anatomical placement, the mechanical properties of the selected graft tissue, its fixation strength, and the biological response to the incorporated graft [2]. These factors directly or indirectly influence the mechanical properties of the knee after ACL reconstruction and impact recovery time, function, and mobility [3-6]. Despite the advances in ACL reconstruction over the decades [2], poor intra-articular graft healing is still a common reason for ACL graft failure. Ligamentization or intra-articular graft healing occurs through a series of biological processes that include early graft healing, proliferation, and ligamentization $[4,7]$. Early graft healing is commonly associated with increases necrosis and a loss of cellularity. This stage is followed by the cellular repopulation of host synovial cells, extracellular matrix synthesis, and revascularization. In the final stage, the healing ACL graft continuously undergoes remodeling and over time regains mechanical strength [8-11]. Research conducted on humans has shown that the process of ligamentization is similar to animals but the remodeling phases is prolonged in humans $[3,4,12,13]$. Animal studies have also shown that ligamentization only restores approximately $50-60 \%$ of the mechanical strength of an intact limb [14-17]. Therefore, alternative strategies to improve ACL reconstruction are needed.

Currently, many ideal graft types exist to reproduce the anatomy and mechanics of a native ACL and include autograft, allograft, or a synthetic graft. The two most commonly used autografts are bone-patellar tendon-bone (BPTB) and hamstrings tendon (HT). BPTB autografts have many advantages including high strength and stiffness, size consistency, and ease of harvesting $[18,19]$. Unfortunately, BPTB autografts are at high risk for donor site morbidity, anterior knee pain, patellar injuries, and loss of function [20]. HT autografts were associated with less donor site morbidity but were originally thought to provide inferior biomechanical properties [21-24]. However, recent biomechanical studies have shown that the HT grafts provide similar or better biomechanical outcomes when compared to the BPTB graft. In a study with 71 patients, patients with HT grafts were found to walk on their knees significantly better than BРTB patients 2 years post procedure [22]. In another study, the HT graft was able to withstand a higher 
load of failure when compared to BPTB $(2422 \mathrm{~N}$ verses $1784 \mathrm{~N}$, respectively [25]. Improvements to $\mathrm{HT}$ autografts such as the quadruple combined semitendinosus tendon and gracilis tendon graft (DSTG) can also improve the biomechanical outcome of the graft. For instance, the DSTG graft's tensile strength has an ultimate load of $4500 \mathrm{~N}$, which is $\sim 2.5 \mathrm{X}$ higher than the ultimate load for a native ACL (1725N) [26]. Other HT modifications such as creating a four-strand graft, by folding two hamstring tendons in half have been tested [1] This modification has been shown to decrease the length of the graft but increases the maximum load and stiffness of the graft. These current advancements have made HT grafts a popular autograft choice during ACL reconstruction..

Other components of ACL reconstruction are important for surgical success including fixation strategy and tendon grafts modifications. Fixation strategies include intratunnel and extratunnel fixation of both femoral and tibia grafts. However, most research data indicates little difference between these methods when using outcome measurement like clinical scores and graft failure as criteria $[27,28]$ Other fixation methods such as the endobutton femoral fixation, the use of bioabsorable or metal interference screws, and the use of a cortical suspension systems exist [29]. In one study, the endobutton fixation method was found to increase stability for the HT graft when compared a BPTB graft fixed with an interference screw [30]. Other data comparing a cortical button fixation with a transfemoral suspensory femoral fixation found little difference between groups when measuring short-term outcomes [31]. Current research suggests that there is no clinical difference between screw fixation and suspensory fixation. Yet, suspensory systems are believed to help with soft tissue graft fixation by limiting graft slippage and providing sufficient fixation strength. Tendon grafts are often modified to increase the tensile strength and stiffness of the graft. Millett et al. [32] found that braiding the tendon grafts decreases the strength and stiffness in BPTB and HT grafts. Grafts can also undergo a process known as whip stitching which converts the flat, harvested tendon into a cylindrical structure, which effectively tubularizes the graft [33]. The graft can then be strung through the tibia and femoral bone tunnel using a femoral suspensory system that prevents graft bunching. Compared to unmodified tendon grafts, whip stitching is thought to increase the tensile strength of the graft and increase the load needed to cause ultimate failure [34]. Though Wang et al showed that this process could decrease the peak tensile load to failure and stiffness of single stranded semitendinosus grafts [35]. The aim of this study was to assess whether whip stitching a quadruple HT graft using a femoral suspensory device increases the tensile strength when the graft is cyclically loaded. We hypothesize that whip stitching a quadruple-strand HT graft will increase stiffness, yield load, and ultimate load when compared to non-whip-stitched graft via mechanical testing.

\section{Methods}

\section{Bovine ACL HT graft preparation}

Bovine extensor tendons $(n=24)$ were obtained from Arthrex, Inc. (Naples, FL) and thawed at room temperature overnight before preparation and testing. During HT ACL graft preparation, all samples were kept at room temperature and moistened periodically with $0.9 \%$ normal saline. The length of each tendon was recorded before testing $(132 \mathrm{~mm} \pm 16)$ and after testing $(142 \mathrm{~mm} \pm 15.5)$. Each tendon was sectioned longitudinally and folded over itself to create a quadruple-strand HT graft around the Endobutton loop (Arthrex, Inc., Naples, FL). Each graft was marked at $40 \mathrm{~mm}$ from the free ends (tibia cortex to the intra-articular surface), 30mm of intraarticular distance, and $20 \mathrm{~mm}$ from the femoral side. Using number 2 Fiber Wire Fiber Loop (Arthrex, Inc., Naples, FL), 5mm gapped whip stitches were performed (spanning $30 \mathrm{~mm}$ of the tibia side) to connect the free ends of each graft to create two independent tails. Next, number 2 FiberWire was used as a FiberLoop to tubularize the graft at $5 \mathrm{~mm}$ gaps for the T-ACL group (Figure 1A), but this step was not completed in the C-ACL group (Figure 2B). Preparation of grafts was alternated between T-ACL and C-ACL and was divided as shown in Table 1 for experimentation. Each quadruple graft was sized with an appropriate sizing guide to accommodate an $8 \mathrm{~mm}$ diameter canal.

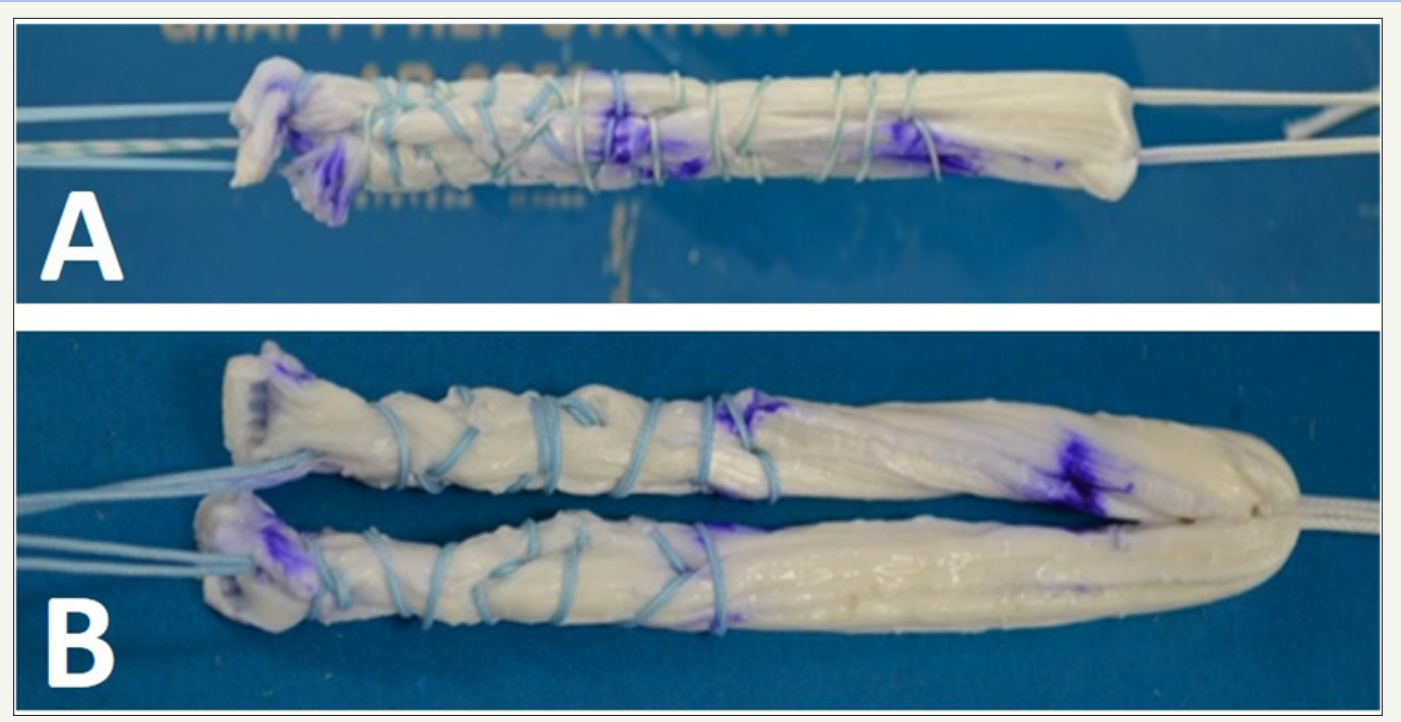

Figure 1: Examples of the Quadruple-Strand ACL Before Mechanically Tested. A) T-ACL B) C-ACL. 
Table 1: Summary of ACL Grafts used in Mechanical Testing Models.

\begin{tabular}{|c|c|c|}
\hline Group Type & $\begin{array}{c}\text { Synthetic Bone Block } \\
\text { Model }\end{array}$ & Porcine MTS Model \\
\hline Tubularized ACL graft & $\mathrm{N}=6$ & $\mathrm{~N}=6$ \\
\hline $\begin{array}{c}\text { Not Tubularized ACL } \\
\text { graft }\end{array}$ & $\mathrm{N}=6$ & $\mathrm{~N}=6$ \\
\hline Total Specimens & 12 & 12 \\
\hline
\end{tabular}

\section{Synthetic bone block model}

A synthetic bone block (SBB) model was completed to optimize the mechanical and outcome parameters for this study. Each SBB model utilized one femoral and one tibia SBB that secured the prepared grafts between them as shown in Figure 2. The SBBs consisted of solid 20lb/ft3 foam for the tibia side (model \#152203, Pacific Research Laboratories, Vashon, WA), and 20lb/ft3 with a $3 \mathrm{~mm}$ thick $40 \mathrm{lb} / \mathrm{ft} 3$ laminate layer for the femur side (model \#1522-65, Pacific Research Laboratories, Vashon, WA). The SBB portion tested 12 grafts total that were divided into 2 groups of six: C-ACL and T-ACL. The preparation of these grafts is described below under graft preparation.

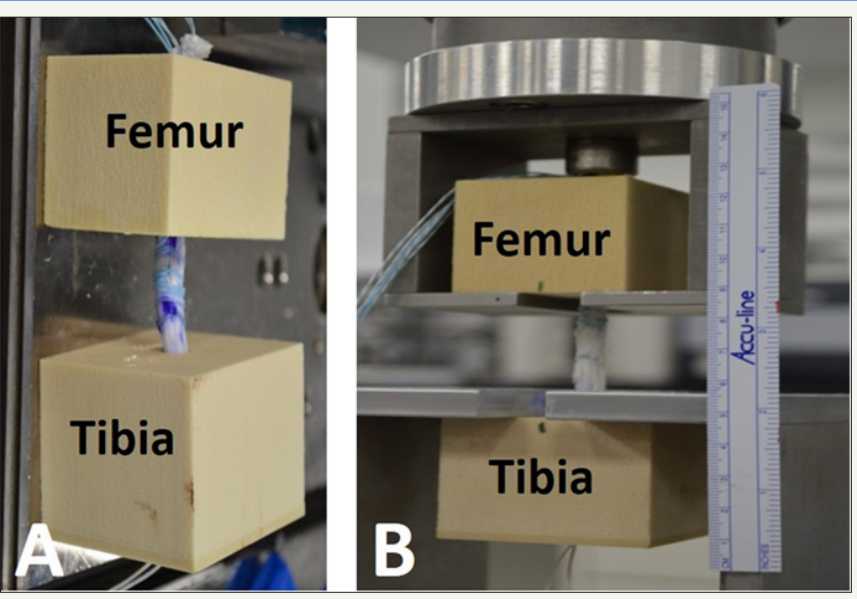

Figure 2: Synthetic Bone Block Model Housed in the Mechanical Testing Device. A) T-ACL graft secured to the bone blocks. B) T-ACL and bone block secured to the mechanical testing machine.

\section{Porcine ACL biomechanical model}

Twelve porcine tibia specimens were obtained from Arthrex, Inc. (Naples, FL), stored in a secured facility, and disposed under approved conditions when the study was complete. The porcine tibias were prepared for the ACL grafts by the same-trained orthopedic resident. During this procedure, the surgeon used a power drill equipped with an $8 \mathrm{~mm}$ drill bit to make a tibia tunnel. Prepared T-ACL and C-ACL grafts were randomly assigned to each tibia and drawn through the tibia tunnel (Figure $3 \mathrm{~A}$ ). The Endobutton was secured over the MTS hooking that simulated femoral fixation, and was secured with an interference screw to finalize the tibia tunnel fixation (9x28mm, Arthrex, Inc., Naples, FL) (Figure 3B \& 3C).
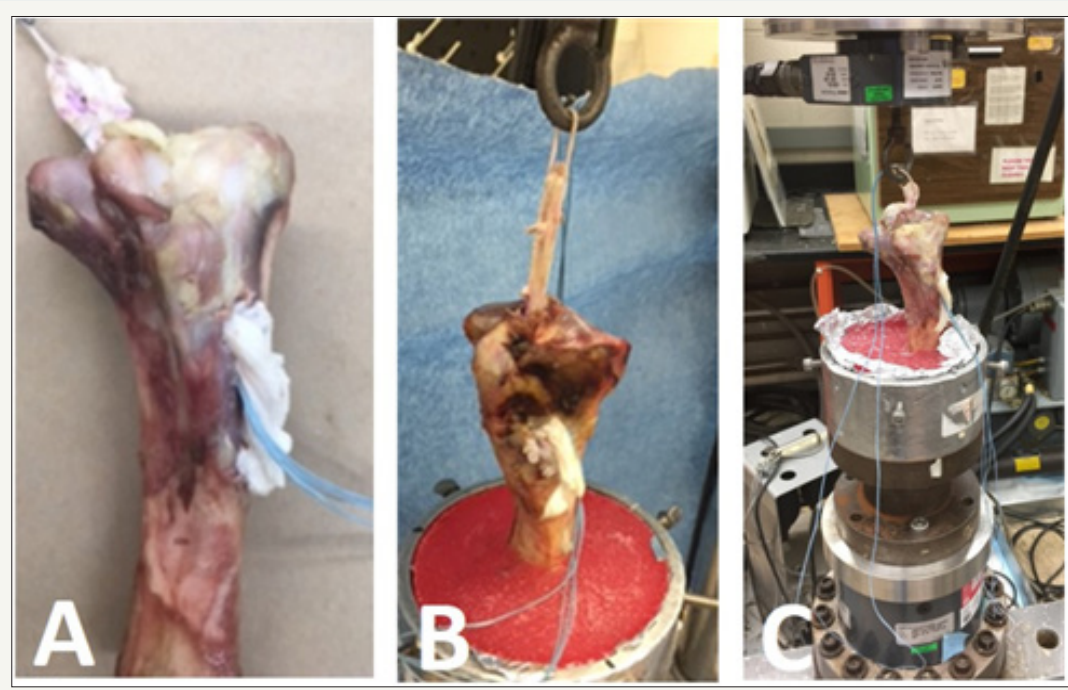

Figure 3:Example of the HT-ACL grafts House in the Mechanical Testing Device. A) T-ACL graft tunneled through the porcine tibia. B) Tibia secured in the mechanical testing aparatus. C) T-ACL secured to machine before testing. 


\section{Mechanical testing}

Methods for biomechanical testing were adopted from the study of Hung et al [36]. For the Porcine ACL Model, the tibias were potted in cylindrical molds of polymethylmethacrylate (PMMA) for gripping in the testing apparatus (Figure 3B). The potting apparatus was secured to the servohydraulic MTS machine using appropriate bolts and fasteners. The grafts were secured to the MTS machine (Minneapolis, MN) through Endobutton connected to a metallic loop (Figure 3C). Samples were pre-loaded between $10 \mathrm{~N}$ and $50 \mathrm{~N}$ for 10 cycles at $0.1 \mathrm{~Hz}$ using the MTS Machine (MTS Systems Corp., Eden Prairie, MN) with a $10 \mathrm{kN}$ load cell Interface, Scottsdale, AZ, USA) in order to ensure all specimens were tested at similar stress-strain conditions. Pre-conditioned specimens

Results

Effect of tubularization via whip stitching on a quadruple-strand hamstring acl using a synthetic bone block model

Table 2: Mechanical Properties for the ACL Graft Groups via Synthetic Bone Block Model.

\begin{tabular}{|c|c|c|c|c|}
\hline & Sample Size(n) & Ultimate Load (N) & Yield Load (N) & $\begin{array}{c}\text { Tendon } \\
\text { Stiffness (N/mm) } \\
\text { Displacement Length } \\
\text { (mm) }\end{array}$ \\
\hline C-ACL Mean & 6 & $762.3 \pm 95.5$ & $658 \pm 93.8$ & $181 \pm 17.6$ \\
\hline T-ACL Mean & 6 & $851.8 \pm 35.5$ & $793 \pm 78.4$ & $173 \pm 6.08$ \\
\hline P Value & & 0.075 & 0.025 & 0.315 \\
\hline
\end{tabular}

The SBB model was used to compare the mechanical properties of the T-ACL and C-ACL groups. The mean ultimate load for the experimental group was $12 \%$ higher when compared to the control but was not found to be significantly different ( $\mathrm{P}=0.075$; Table 2). When yield load was compared between the groups the T-ACL and immediately underwent cyclic/fatigue testing between $50 \mathrm{~N}$ and $250 \mathrm{~N}$ for 500 cycles at $1 \mathrm{~Hz}$. Following cyclic loading, a pull to failure was conducted at $20 \mathrm{~mm} / \mathrm{min}$.

\section{Data analysis}

The slope of the liner region of the load displacement curve was used to determine stiffness $(\mathrm{N} / \mathrm{mm})$ of the ACL. Ultimate load $(\mathrm{N})$ was defined as the maximum load before a sudden drop in force. withstand before becoming permanently deformed. T-tests were used to determine significance for ultimate load, yield load, stiffness, and tendon displacement. Statistical analysis was completed using SigmaPlot Version 12 software. Yield Load (N) was defined as the stress level the tendon could

\section{Effect of tubularization via whip stitching on a quadruple-strand hamstring acl using a porcine model}

Table 3: Mechanical Properties for the ACL Graft Groups via Porcine Model.

\begin{tabular}{|c|c|c|c|c|c|}
\hline & Sample Size(n) & Ultimate Load (N) & Yield Load (N) & Stiffness (N/mm) & $\begin{array}{c}\text { Tendon } \\
\text { Displacement } \\
\text { Length(mm) }\end{array}$ \\
\hline C-ACL Mean & 6 & $569.37 \pm 239.09$ & $180.20 \pm 150.10$ & $155.09 \pm 74.98$ & $13.00 \pm 4.77$ \\
\hline T-ACL Mean & 6 & $457.64 \pm 173.91$ & $268.52 \pm 70.75$ & $285.70 \pm 69.67$ & $9.71 \pm 2.56$ \\
\hline P Value & & 0.0351 & 0.26 & 0.015 & 0.142 \\
\hline
\end{tabular}

The effect of tubularization on a quadruple-strand hamstring ACL was compared using porcine tibia as a model. When ultimate load was compared between T-ACL and C-ACL no significant difference was found (Table 3). The T-ACL group was found to have a 1.5-fold higher yield strength when compared to C-ACL but was not significantly different (Figure 4). When stiffness was compared, the T-ACL group was found to be $83 \%$ greater than the C-ACL group (Table 3, $\mathrm{P}=0.015$ ). Additionally, no significant difference was found when the length of displacement was compared between groups (Table 3; $\mathrm{P}=0.142$ ).

\section{Discussion}

The aim of this study was to assess whether whip-stitching a quadruple hamstring allograft in a femoral suspensory model increases the biomechanical properties of the ACL graft. Our results show a statistically significant increase in stiffness in the T-ACL group when using the porcine model and significant increase in
C-ACL groups was found to be statistically greater by $20 \%(P=0.025$; Table 1). No significant difference was found between the groups when stiffness and displacement were compared. yield strength when using the SBB model. Therefore, tubularization via whip stitching may improve the ligamentization process by increase the ACL graft strength and preventing early ACL graft failure.

To date several suturing preparations have been utilized for graft preparation including whip stitching, krackow stitch, and the baseball stitch [37]. It is common during surgery to suture the free ends of the tendon to aid in harvesting and to apply tension to the graft during final fixation. The use of the whip stitching has been shown to withstand higher ultimate loads then a commonly used utility suture [38]. Whip stitching increases the overall diameter of the graft and creates a uniform structure among the four strands, its structure is thought to increase stiffness and prevent deformation of the graft. Our mechanical data obtained with the SBB model demonstrates that T-ACL requires a higher force to deform the ACL and our porcine model shows a similar trend although not 
statistically significant. Other data has shown that a locking whip stitch has similar biomechanical properties when compared to the kratckow stitch. Both stitch type requires similar forces to alter or weaken the mechanical integrity of the graft as measured by ultimate load or tendon elongation after cyclic loading when compared to traditional stitched [39]. One possibly for enhanced mechanical properties is this quadruple-strand whip stitch structure could reduce "the bungee effect". The bungee effect occurs when there is motion along the graft in the longitudinal axis that impairs the biologic incorporation of the graft and instead causes tunnel within the bone to widening [1]. It also possible that this fixation method reduces excessive transverse motion of the graft during flexion and extension of the knee or the additional whipstitch creates more frictional force at the screw graft interface $[1,25,35,38]$. However, Hong et al. [39] demonstrated that the number of suture throws does not have an effect on mechanical properties but very few studies have been completed to support this result. Our study demonstrated that whip stitching in combination with a femoral suspensory fixation enhances yield strength in the SBB model and stiffness in the porcine model which to our knowledge has not been tested.

Limitations of the study are that this biomechanical study since porcine tendons was used in place of human tendons. However, porcine tendons have been established as a reproducible model for ex vivo testing. In addition, the sample size of 6 specimens for each group may have been under powered, however this sample size corresponds to similar biomechanical studies. Furthermore, the non-absorbable intra-articular suture could lead to a sterile intra-articular reaction which may increase the stiffness and yield strength but lead to a suboptimal clinical environment. Additionally, extra suture throws may over constrain the knee joint in a clinical setting and create less optimal soft tissue laxity for normal knee kinematics.

\section{Conclusion}

Approximately 100,000 primary ACL reconstructive surgeries are performed in the United States every year with an everincreasing ACL revision rate of 3,000 to 10,000 per year. Many surgeons are now opting to use allografts due to easier accessibility, better-quality control, and less overall morbidity compared to autografts. However, biologic incorporation of grafts, especially allografts, can be a cause of failure. Therefore, augmentation strategies have been adopted to help protect the graft during the ligamentization process. To be an ideal ACL augmentation tool, the device needs to be strong, low-profile, and non-reactive. Future directions will be to incorporate this technique into an animal model to assess for intra-articular reaction with the nonabsorbable suture and to compare to an absorbable suture at different time points. This ACL reconstruction augmentation may allow for accelerated rehabilitation, graft protection during healing process, reduce cost, and cause uniform tensioning of the graft. Additional whip-stitching may act as a load sharing technique to increase stiffness, yield strength in suspensory fixation quadruple stranded hamstring ACL graft.

\section{References}

1. Bach B, Provencher MT (2010) How to Get it Right the First Time asn What to Do if It Fails. Journal of Sports Science Medicine 9(3): 527.

2. Janssen RP, Scheffler SU, Intra-articular remodelling of hamstring tendon grafts after anterior cruciate ligament reconstruction. Knee Surg Sports Traumatol Arthrosc 22(9): 2102-2108.

3. Falconiero RP, DiStefano VJ, Cook TM (1998) Revascularization and ligamentization of autogenous anterior cruciate ligament grafts in humans. Arthroscopy 14(2): 197-205.

4. Janssen RP, van der Wijk J, Fiedler A, Schmidt T, Sala HA (2011) Remodelling of human hamstring autografts after anterior cruciate ligament reconstruction. Knee Surg Sports Traumatol Arthrosc 19(8): 1299-306.

5. Marumo K, Saito M, Yamagishi T, Fujii K (2005) The "ligamentization" process in human anterior cruciate ligament reconstruction with autogenous patellar and hamstring tendons: a biochemical study. Am J Sports Med 33(8): 1166-1173.

6. Mayr HO, Stoehr A, Dietrich M, von Eisenhart-Rothe R, Hube R (2012) Graft-dependent differences in the ligamentization process of anterior cruciate ligament grafts in a sheep trial. Knee Surg Sports Traumatol Arthrosc 20(5): 947-956.

7. Amiel D, Kleiner JB, Roux RD, Harwood FL, Akeson WH (1986) The phenomenon of "ligamentization": anterior cruciate ligament reconstruction with autogenous patellar tendon. J Orthop Res 4(2): 162172 .

8. Papalia R, Franceschi F, Vasta S, Di Martino A, Maffulli N, et al. (2012) Sparing the anterior cruciate ligament remnant: is it worth the hassle? Br Med Bull 104: 91-111.

9. Scheffler SU1, Scherler J, Pruss A, von Versen R, Weiler A (2005) Biomechanical comparison of human bone-patellar tendon-bone grafts after sterilization with peracetic acid ethanol. Cell Tissue Bank 6(2): 109-115.

10. Unterhauser FN, Bail HJ, Höher J, Haas NP, Weiler A (2003) Endoligamentous revascularization of an anterior cruciate ligament graft. Clin Orthop Relat Res 414: 276-288.

11. Weiler A, Peters G, Mäurer J, Unterhauser FN, Südkamp NP (2001) Biomechanical properties and vascularity of an anterior cruciate ligament graft can be predicted by contrast-enhanced magnetic resonance imaging. A two-year study in sheep. Am J Sports Med 29(6): 751-761.

12. Claes S, Verdonk P, Forsyth R, Bellemans J (2011) The "ligamentization" process in anterior cruciate ligament reconstruction: what happens to the human graft? A systematic review of the literature. Am J Sports Med 39(11): 2476-2483.

13. Lane JG, McFadden P, Bowden K, Amiel D (1993) The ligamentization process: a 4 year case study following ACL reconstruction with a semitendinosis graft. Arthroscopy 9(2): 149-153.

14. Beynnon BD, Uh BS, Johnson RJ, Abate JA, Nichols CE (2005) Rehabilitation after anterior cruciate ligament reconstruction: a prospective, randomized, double-blind comparison of programs administered over 2 different time intervals. Am J Sports Med 33(3): 347-359.

15. Goradia VK, Rochat MC, Grana WA, Rohrer MD, Prasad HS (2000) Tendon-to-bone healing of a semitendinosus tendon autograft used for ACL reconstruction in a sheep model. Am J Knee Surg 13(3): 143-151.

16. Jackson JR, Minton JA, Ho ML, Wei N, Winkler JD (1997) Expression of vascular endothelial growth factor in synovial fibroblasts is induced by hypoxia and interleukin 1beta. J Rheumatol 24(7): 1253-1259.

17. Ng GY, Oakes BW, Deacon OW, McLean ID, Lampard D (1995) Biomechanics of patellar tendon autograft for reconstruction of the 
anterior cruciate ligament in the goat: three-year study. J Orthop Res 13(4): 602-608.

18. Dheerendra SK, Khan WS, Singhal R, Shivarathre DG, Pydisetty R, et al. (2012) Anterior cruciate ligament graft choices: a review of current concepts. Open Orthop J 6: 281-286.

19. Fineberg MS1, Zarins B, Sherman OH (2000) Practical considerations in anterior cruciate ligament replacement surgery. Arthroscopy 16(7): 715-724.

20. Busam ML, Provencher MT, Bach BR Jr (2008) Complications of anterior cruciate ligament reconstruction with bone-patellar tendon-bone constructs: care and prevention. Am J Sports Med 36(2): 379-394

21. Aglietti P, Giron F, Buzzi R, Biddau F, Sasso F (2004) Anterior cruciate ligament reconstruction: bone-patellar tendon-bone compared with double semitendinosus and gracilis tendon grafts. A prospective, randomized clinical trial. J Bone Joint Surg Am 86-A(10): 2143-2155.

22. Ejerhed L, Kartus J, Sernert N, Köhler K, Karlsson J (2003) Patellar tendon or semitendinosus tendon autografts for anterior cruciate ligament reconstruction? A prospective randomized study with a twoyear follow-up. Am J Sports Med 31(1): 19-25.

23. Feller JA, Webster KE (2003) A randomized comparison of patellar tendon and hamstring tendon anterior cruciate ligament reconstruction. Am J Sports Med 31(4): 564-573.

24. Laxdal G, Kartus J, Hansson L, Heidvall M, Ejerhed L, et al. (2005) A prospective randomized comparison of bone-patellar tendon-bone and hamstring grafts for anterior cruciate ligament reconstruction. Arthroscopy 21(1): 34-42.

25. Wilson TW, Zafuta MP, Zobitz M (1999) A biomechanical analysis of matched bone-patellar tendon-bone and double-looped semitendinosus and gracilis tendon grafts. Am J Sports Med 27(2): 202-207.

26. Hamner DL, Brown CH Jr, Steiner ME, Hecker AT, Hayes WC (1999) Hamstring tendon grafts for reconstruction of the anterior cruciate ligament: biomechanical evaluation of the use of multiple strands and tensioning techniques. J Bone Joint Surg Am 81(4): 549-557.

27. Han DL, Nyland J, Kendzior M, Nawab A, Caborn DN (2012) Intratunnel versus extratunnel fixation of hamstring autograft for anterior cruciate ligament reconstruction. Arthroscopy 28(10): 1555-1566.

28. Ilahi OA, Nolla JM, Ho DM (2009) Intra-tunnel fixation versus extratunnel fixation of hamstring anterior cruciate ligament reconstruction: a meta-analysis. J Knee Surg 22(2): 120-129.

Creative Commons Attribution 4.0 International License

For possible submissions Click Here

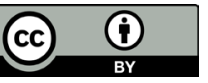

Submit Article
29. Anderson MJ, Browning WM, Urband CE, Kluczynski MA, Bisson LJ (2016) A Systematic Summary of Systematic Reviews on the Topic of the Anterior Cruciate Ligament. Orthop J Sports Med 4(3): 2325967116634074

30. Prodromos CC, Joyce BT, Shi K, Keller BL (2005) A meta-analysis of stability after anterior cruciate ligament reconstruction as a function of hamstring versus patellar tendon graft and fixation type. Arthroscopy 21(10): 1202

31. Saccomanno MF, Shin JJ, Mascarenhas R, Haro M, Verma NN, et al. (2014) Clinical and functional outcomes after anterior cruciate ligament reconstruction using cortical button fixation versus transfemoral suspensory fixation: a systematic review of randomized controlled trials. Arthroscopy 30(11): 1491-1498.

32. Millett PJ, Miller BS, Close M, Sterett WI, Walsh W, et al. (2003) Effects of braiding on tensile properties of four-strand human hamstring tendon grafts. Am J Sports Med 31(5): 714-717.

33. Krappinger D, Kralinger FS, El Attal R, Hackl W, Haid C (2007) Modified Prusik knot versus whipstitch technique for soft tissue fixation in anterior cruciate ligament reconstruction: a biomechanical analysis. Knee Surg Sports Traumatol Arthrosc 15(4): 418-423.

34. Corry IS, Webb JM, Clingeleffer AJ, Pinczewski LA (1999) Arthroscopic reconstruction of the anterior cruciate ligament. A comparison of patellar tendon autograft and four-strand hamstring tendon autograft. Am J Sports Med 27(4): 444-454.

35. Wang RY, Arciero RA, Obopilwe E, Mazzocca AD (2010) A comparison of structural and mechanical properties of tubularized and native semitendinosus graft. Am J Sports Med 38(6): 1246-1249.

36. Chu-Chih Hung, Chan-Tsung Yang, Cheng-Kung C Heng, Chih-Hwa Chen, Yu-Shu Lai (2014) Interference screw versus Endoscrew fixation for anterior cruciate ligament reconstruction: A biomechanical comparative study in sawbones and porcine knees. Journal of Orthopaedic Translation 2(2): $82-90$

37. Camarda L, Giambartino S, Lauria M, Saporito M, Triolo V, et al. (2016) Surgical time for graft preparation using different suture techniques. Muscles Ligaments Tendons J 6(2): 236-240.

38. da Assunção RE, Haddad R, Bruce WJ, Walker P, Walsh WR (2013) Whip stitch versus grasping suture for tendon autograft. Eur J Orthop Surg Traumatol 23(1): 105-109.

39. Hong CK, Lin CL, Chang CH, Jou IM, Su WR Effect of the number of suture throws on the biomechanical characteristics of the suture-tendon construct. Arthroscopy 30(12): 1609-1615.

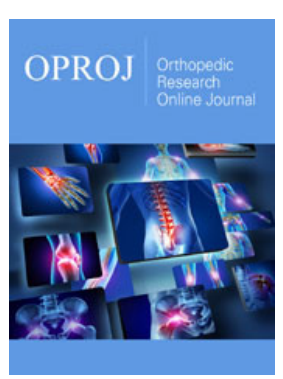

Orthopedic Research Online Journal

Benefits of Publishing with us

- High-level peer review and editorial services

- Freely accessible online immediately upon publication

- Authors retain the copyright to their work

- Licensing it under a Creative Commons license

- Visibility through different online platforms 